\title{
Axis Measurements, Field Quality and Quench Performance of the First LHC Short Straight Sections
}

\author{
S. Sanfilippo, N. Smirnov, P. Schnizer, N. Sammut, P. Pugnat, L. Bottura, A. Siemko, M. Calvi, V. Chohan, \\ A. Stafiniak, L. Walckiers, P. Hagen, E. Todesco, T. Tortschanoff, F. Simon, and M. Durante
}

\begin{abstract}
The series testing at $1.9 \mathrm{~K}$ of the 360 Short Straight Sections (SSS) for the Large Hadron Collider have started at CERN in September 2003. The SSS contain the lattice quadrupoles and correction magnets in a common cryostat. The lattice quadrupoles feature two collared coils with $56 \mathrm{~mm}$ bore assembled in a common yoke. The coils are wound in two-layers from 15.1 mm wide NbTi cable, insulated with polyimide tape. The paper reviews the main test results performed in superfluid helium. The magnetic field and magnetic center position of the quadrupoles and associated correctors were measured with two independent systems, namely an automated scanner and a single stretched wire technique. The quench training, the field quality and the magnetic alignment measurements are presented and discussed in terms of the specifications and expected performances of these magnets in the LHC. We discuss in detail the field quality in terms of multipole errors measured at injection and nominal field and decomposed into geometric and persistent current magnetization errors. Warm/cold correlation for the geometric multipoles and the magnetic axis is also presented.
\end{abstract}

Index Terms-Field quality, quench training, superconducting accelerator magnets.

\section{INTRODUCTION}

$\mathbf{T}$ HE Large Hadron Collider (LHC), now under construction at CERN will contain 360 lattice quadrupoles (MQ) assembled in arc Short Straight Sections (SSS) of 61 different types [1]. Arc SSS will operate at $1.9 \mathrm{~K}$ and their quadrupole at nominal field gradient of $223 \mathrm{~T} / \mathrm{m}$.

The development of the lattice quadrupole magnets started in 1989 in collaboration with the CEA-Saclay for the design of the cold mass and with the CNRS laboratory of Orsay in France for the design of the cryostat. These collaborations resulted in a successful construction and testing of 3 prototypes [2]-[4]. The 360 fully assembled cold masses needed for the LHC were ordered with the German firm ACCEL Instrument in July 2000, CEA and CERN ensuring the technology transfer and the follow up of the series fabrication [5]. The cold testing of the series SSS started in September 2003 at the CERN Superconducting Magnet Test Plant (SMTP) [6].

In this paper, the main test results of the twenty series SSS tested up to end of August 2004 will be reviewed. The results of the electrical tests, the quench training performance, field

Manuscript received October 4, 2004.

S. Sanfilippo, N. Smirnov, N. Sammut, P. Pugnat, L. Bottura, A. Siemko, M. Calvi, V. Chohan, A. Stafiniak, L. Walckiers, P. Hagen, E. Todesco, and T. Tortschanoff are with CERN, Geneva, Switzerland (e-mail: stephane. sanfilippo@cern.ch).

P. Schnizer is with the GSI, Germany.

F. Simon and M. Durante are with CEA-Saclay, France.

Digital Object Identifier 10.1109/TASC.2005.849504 quality and magnetic axis measurements will be discussed in terms of the design parameters.

\section{Magnet Design Features}

The design of the arc short straight sections is described in detail in [2]-[5]. Only the main design features will be recalled here in view of the discussion of the test results. The lattice quadrupole features two independent apertures with $56 \mathrm{~mm}$ bore assembled into a common yoke separated by a distance of $194 \mathrm{~mm}$ at $1.9 \mathrm{~K}$. The two layers of the quadrupole coils are wound with a $15.1 \mathrm{~mm}$ wide NbTi Rutherford cable made of 36 strands of $0.825 \mathrm{~mm}$ diameter coming from three manufacturers. The magnetization of the strands is measured at CERN at a field of $0.5 \mathrm{~T}$ in a $1.9 \mathrm{~K}$ helium bath. The maximum values specified for the LHC production are $23 \mathrm{mT}$ with the control limits of $\pm 4.5 \%$ around their average value. The main quadrupole is designed to work at an injection field gradient of $14.5 \mathrm{~T} / \mathrm{m}(0.45 \mathrm{TeV}$ beam energy) and at a nominal field gradient of $223 \mathrm{~T} / \mathrm{m}$ ( $7 \mathrm{TeV}$ beam energy) corresponding respectively to currents of $763 \mathrm{~A}$ and $11870 \mathrm{~A}$. It reaches a gradient of $241 \mathrm{~T} / \mathrm{m}$ with a peak field in the coil of $7.5 \mathrm{~T}$ at ultimate machine performance ( $9 \mathrm{~T}$ dipole field).

Each coil is independently collared with $2 \mathrm{~mm}$-thick non magnetic austenitic steel collars. The two separated apertures are then assembled in a laminated yoke.

The SSS cold mass consists of a $5.3 \mathrm{~m}$ long inertia tube into which the MQ and correctors are precisely aligned. The correctors are an octupole MO, tuning quadrupole MQT or skew quadrupole correctors MQS on one end and a combined sextupole-dipole correctors MSCB on the other end.

\section{Performances of the First Series Short Straight SECTION}

\section{A. Test Procedure}

The SSS are cold tested at CERN in the SMTP, consisting of 12 tests stands and the necessary cryogenic infrastructures to perform the tests of all the main ring magnets within the allocated time [6]. The cold tests are used to qualify the cryogenic and vacuum integrity. Electrical tests are executed after each critical phase and power tests are carried out aiming at the qualification of the magnets in terms of the number of training quenches necessary to reach the nominal $(223 \mathrm{~T} / \mathrm{m})$ and the ultimate $(241 \mathrm{~T} / \mathrm{m})$ field gradient levels.

The field quality is measured in machine operating conditions after the training of the MQ. The beam screens are not mounted in the magnet apertures to allow the insertion of the 


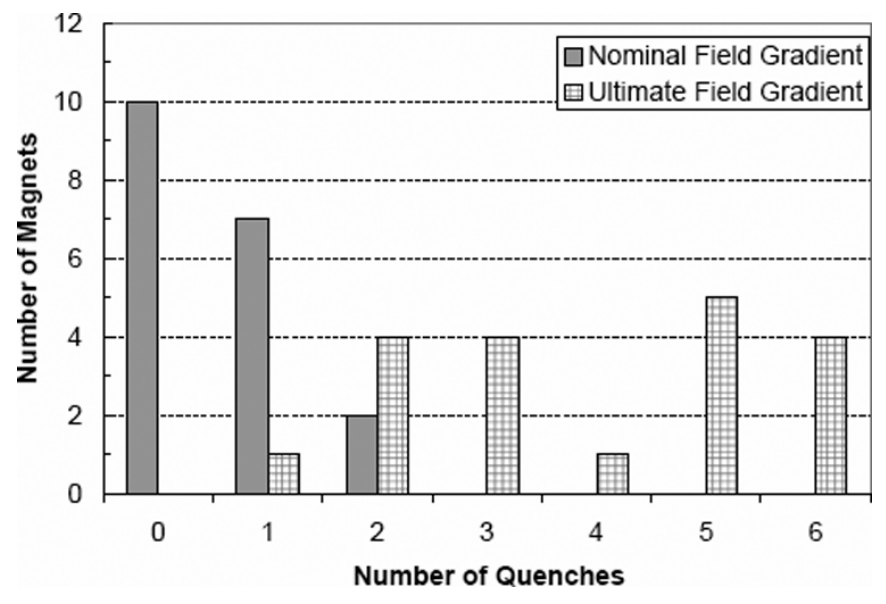

Fig. 1. Histogram of the number of quenches to reach the nominal $(223 \mathrm{~T} / \mathrm{m})$ and ultimate $(241 \mathrm{~T} / \mathrm{m})$ field gradient.

so-called "anticryostats" for the use of magnetic systems like the automated scanner [7], the single stretched wire (SSW) [8] and the $10-\mathrm{m}$ long shafts based on the $15-\mathrm{m}$ long ones used for the dipoles [9].

The position of the magnetic axis of the MQ and of the correctors with respect to an external frame are determined at $1.9 \mathrm{~K}$ and in warm conditions $(300 \mathrm{~K})$ using the SSW or the automated scanner. The MQ axis is measured with $0.1 \mathrm{~mm}$ accuracy at the intermediate current of $5 \mathrm{kA}$ at cold and at $20 \mathrm{~A} \mathrm{DC}$ current at room temperature. The stability of the magnetic axis versus current has been investigated on a few MQs showing an acceptable deviation below $0.1 \mathrm{~mm}$.

The SSW system localizes the correctors with the so called "rotating wire technique", at the DC nominal current in cryogenic condition and with an $\mathrm{AC}$ excitation at room temperature. The reproducibility of the corrector axis measurement is with both systems in the range of, $0.1 \mathrm{~mm}$ and $0.2 \mathrm{~mm}$, respectively at cryogenic and room temperature. The SSW uses AC excitation at room temperature of respectively $5 \mathrm{~A}$ and $1 \mathrm{~A}$ for the $\mathrm{MQ}$ and of the correctors. The SSW system is faster and simpler to use and will be employed for series measurement.

\section{Electrical and Training Quench Performance}

The electrical tests consist mainly of DC resistance measurements to check the integrity of the magnet instrumentation and of insulation measurements performed at high voltage for the MQ and the correctors versus ground and between each other. For the standard quench training test, the quench current is reached with a nominal ramp rate of $10 \mathrm{~A} / \mathrm{s}$. The histogram in Fig. 1 shows the number of quenches to reach the nominal and the ultimate field gradient for all the MQs tested to date. The magnets display satisfactory quench performance, all quadrupoles reaching the nominal gradient of $223 \mathrm{~T} / \mathrm{m}$ after at most the 2nd quench and the ultimate gradient after a maximum of 6 quenches. Four quadrupoles were tested after a thermal cycle from $1.8 \mathrm{~K}$ to room temperature and back to $1.8 \mathrm{~K}$ and showed a good memory effect with no quench recorded below $236 \mathrm{~T} / \mathrm{m}$.

Two SSS were registered as nonconforming out of the 20 cold tested. One could not be tested in cryogenic conditions due to

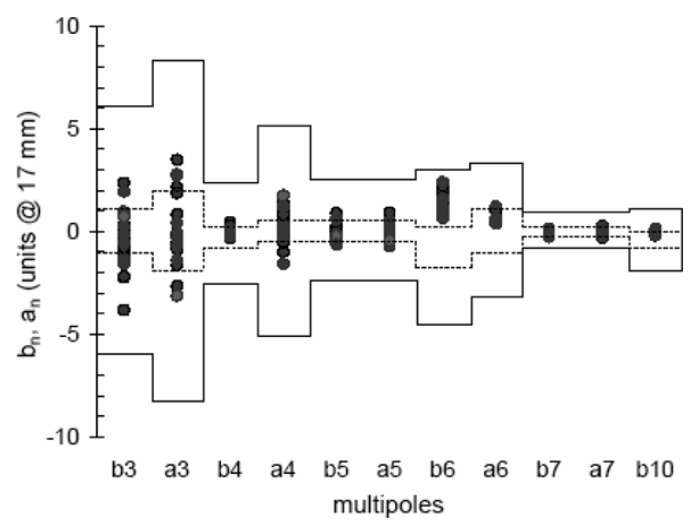

Fig. 2. Field quality at injection field. The dotted lines display the upper and lower limits for the systematic value and the continuous line the limits for an individual quadrupole.

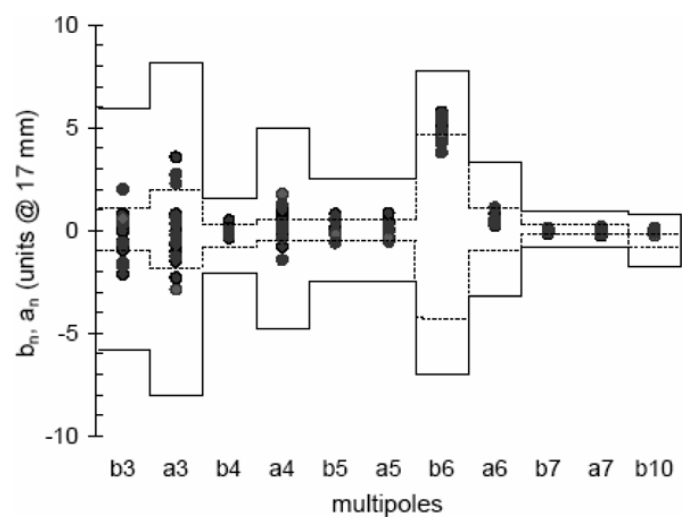

Fig. 3. Field quality at nominal field. The dotted lines display the upper and lower limits for the systematic value and the continuous line the limits for an individual quadrupole.

a failure of the protection quench heaters. In the second one the insulation of the corrector MQT to ground did not pass the specified voltage of $1.5 \mathrm{kV}$.

\section{MAGNETIC Field QuALiTy}

\section{A. Field Quality at Injection and Nominal Current}

So far, the field quality of seven short straight sections (fourteen apertures) has been measured in cold conditions. The higher order multipole components at injection and at nominal field of the measured MQs are summarized in Figs. 2 and 3. Normal and skew field multipoles, $b_{n}$ and $a_{n}$ respectively, are normalized to the main quadrupole field $(\mathrm{n}=2)$, scaled by a factor $10^{4}$ and expressed at a reference radius of $17 \mathrm{~mm}$. The multipoles plotted are integrated over the magnet length, including ends, and are compared to the maximum bounds for the average (the systematic) and for the spread allowed by the LHC operation (i.e. the latter being the limits for an individual quadrupole) [10].

At injection and nominal fields, most integrated multipoles are within the allowable limits. The large normal do-decapole, values at injection and nominal fields $\left(b_{6}\right)$, are inherent to the coil geometry of the first series quadrupoles. The observed discrepancy of about +1.5 units can be explained by the consequence of an additional compression of the coils by the col- 


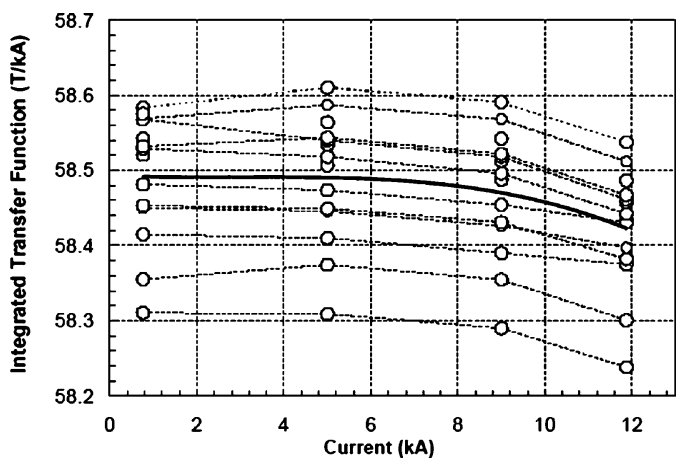

Fig. 4. Integrated transfer functions for the $14 \mathrm{MQ}$ apertures as a function of the current. The bold continuous line displays the average transfer function.

TABLE I

INTEGRATED TRANSFER FunCtion (AVERAGE, SPREAD) AT INJECTION AND NOMINAL CURRENT VERSUS THE SPREAD EXPECTED BY BEAM DYNAMICS

\begin{tabular}{cccc}
\hline \hline Current level. & $\begin{array}{c}\text { Average } \\
\text { measured } \\
\text { T/kA }\end{array}$ & $\begin{array}{c}\sigma \\
\text { measured } \\
\text { (units) }\end{array}$ & $\begin{array}{c}\sigma \\
\text { beam dynamics } \\
\text { (units) }\end{array}$ \\
\hline Injection (763 A) & 58.499 & 14.9 & 10 \\
Geometric(5 kA) & 58.496 & 14.9 & 10 \\
Nominal (11870 A) & 58.422 & 14.5 & 10 \\
\hline \hline
\end{tabular}

lars [11], not taken into account in the first definition of the coil cross-section. A corrective action has been agreed between CERN and CEA, consisting in adding in the mid-plane an additional insulation sheet of $0.125 \mathrm{~mm}$.

\section{B. Integrated Transfer Function of the Gradient}

The integrated transfer function (TF) of the field gradient (ratio of integrated quadrupole to operating current) is plotted as function of the current in Fig. 4.

The average and the standard deviation calculated at injection, at $5 \mathrm{kA}$ and at nominal current are presented in Table I. These latter are compared to the specified r.m.s. imposed by the beam dynamics [10].

The saturation of the ferromagnetic yoke results in a reduction of the transfer function at nominal field by approximately 13 units with respect to the geometric value evaluated at $5 \mathrm{kA}$.

The spread observed among the seven magnets tested amounts to 15 units, above the limit of the specified r.m.s. value of 10 units. This can generate a $\beta$ beating out of the tolerance budget.

\section{Warm-Cold Correlation}

Room temperature measurements are performed at the quadrupole manufacturer to evaluate the geometric field errors. Fig. 5 shows the correlation of the transfer function of the gradient (TF) and of the geometric part of the do-decapole $\left(b_{6}\right)$ between room temperature and cryogenic temperature measurements. The geometric contribution is computed as the average of the measured values on the ramp up and ramp down powering branches at $5 \mathrm{kA}$. The warm measurements are performed at a current of $12 \mathrm{~A}$ with positive and negative currents to eliminate the residual magnetization. As displayed in Fig. 5, the correlation with the slope one is very good and a small offset is observed for $b_{6}$.
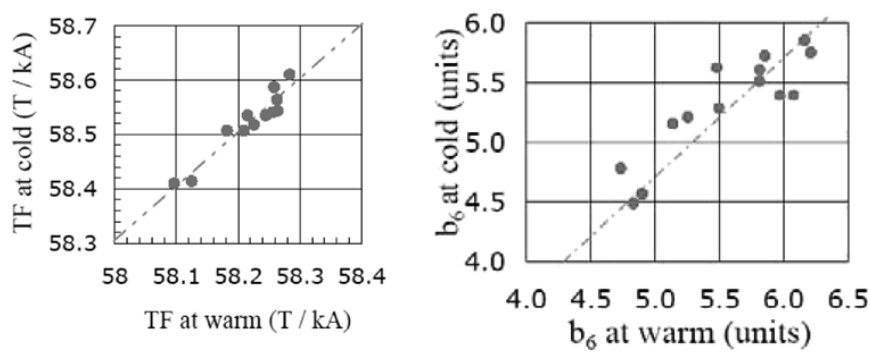

Fig. 5. Scatter plot of the transfer function of the gradient (left) and of the do-decapole (right) measured in warm and cold conditions (geometric part only). Experimental points are compared with a linear fit with a slope 1 related to the ideal correlation.

TABLE II

STANDARD DEVIATION AND OFFSET OF THE CORRELATION BETWEEN MEASUREMENTS OF GEOMETRIC FIELD QUALITY AT $300 \mathrm{~K}$ AND AT $1.9 \mathrm{~K}$ (IN UNITS AT $17 \mathrm{~mm}$ )

\begin{tabular}{ccccc}
\hline \hline $\begin{array}{c}\text { Multipole } \\
\text { order }\end{array}$ & $\Delta_{\text {offset }}$ & $\sigma_{\text {warm-cold }}$ & $\begin{array}{c}\sigma_{\text {beam }} \\
\text { dynamics } \\
\text { injection }\end{array}$ & $\begin{array}{c}\sigma_{\text {beam }} \\
\text { dynamics } \\
\text { nominal }\end{array}$ \\
\hline TF & 50 & 6.50 & 10 & 10 \\
$\mathrm{~b}_{3}$ & 0.07 & 0.38 & 1.35 & 1.30 \\
$\mathrm{a}_{3}$ & 0.08 & 0.33 & 1.44 & 1.40 \\
$\mathrm{~b}_{4}$ & -0.51 & 0.36 & 0.30 & 0.30 \\
$\mathrm{a}_{4}$ & -0.1 & 0.60 & 0.90 & 1.30 \\
$\mathrm{~b}_{6}$ & -0.27 & 0.24 & 0.60 & 0.60 \\
$\mathrm{~b}_{10}$ & -0.04 & 0.04 & 0.35 & 0.35 \\
\hline \hline
\end{tabular}

Table II shows the offset between cold and warm data $\left(\Delta_{\text {offset }}\right)$ and the standard deviation of the warm-cold correlation $\left(\sigma_{\text {warm-cold }}\right)$ for the transfer function and for the most critical multipoles. The standard deviation corresponds to the spread around the ideal correlation line.

For the critical parameters $\mathrm{TF}$ and $\mathrm{b}_{6}$, the obtained offsets are in accordance with the expectations. Taking into account the change of the magnet geometry induced by thermal contraction, the computed warm-cold offsets are 49 units and -0.7 units respectively for the transfer function and the $\mathrm{b}_{6}$ [12]. Apart from $\mathrm{b}_{4}, \sigma_{\mathrm{warm}-\text { cold }}$ is about two times lower or more than the standard deviation allowed by the beam dynamics at injection and nominal field [10]. This confirms that measurements at $300 \mathrm{~K}$ are an effective mean to control the geometric harmonics in operating conditions. The origin of the $\sigma_{\text {warm-cold }}$ observed for the $\mathrm{b}_{4}$ is under investigation.

\section{Magnetization Effects of Multipoles at Injection}

The magnetization associated with persistent currents in the NbTi superconducting filaments changes the allowed harmonics $\left(b_{2}, b_{6}\right.$ and $\left.b_{10}\right)$ especially at low fields. This contribution to the field error is defined as the difference between the multipole value measured once the injection current is reached and its geometric component. The magnetization effect on $b_{6}$ and $b_{10}$ was found to be in agreement with the expectations. Average field changes of -3.48 units and 0.06 units were measured in accordance with the calculated contributions of -3.48 units and 0.1 units based on strands measurements [13]. In the case of $b_{2}$, the magnetization effect did not match with the expectations with an average change of 0.38 units as compared to the -4.39 units expected [13]. The random component for $b_{2}$ was also larger than predicted with an r.m.s. of 2.6 units versus the 0.6 units 

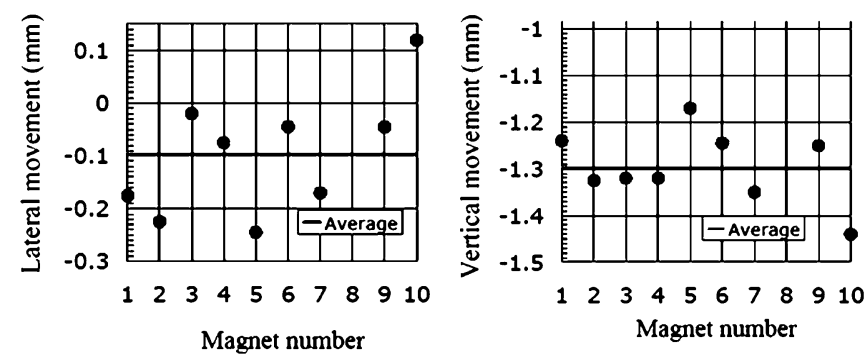

Fig. 6. Lateral (left) and vertical (right) movements of the SSS during the cool down. The magnet number 8 was incorrectly measured and the results removed. The horizontal grey straight line reflects the average in both cases.

expected. This discrepancy is supposed to come from a higher permeability than specified of the stainless steel collars.

\section{MAGNETIC AXIS}

The first seven SSS were tested with the SSW and the automated scanner showing a typical agreement of the axis measurement of $0.2 \mathrm{~mm}$. The axes of $10 \mathrm{SSS}$ have been measured at both room and cryogenic temperatures. The cool down effect was estimated assuming that the SSS external fiducials are stable during the temperature change. They usually move by less than $0.1 \mathrm{~mm}$ with respect to the reference network. The amplitude of the cold mass movements during the cool down in the lateral and vertical directions are presented in Fig. 6 .

The measurements show that:

- in vertical direction the MQ axes moves down by $1.3 \mathrm{~mm}$ as expected (movement of the support post during the cool down), with a standard deviation of $0.1 \mathrm{~mm}$

- $\quad$ laterally the MQ moves unexpectedly toward the external side by $0.1 \mathrm{~mm}$ with a standard deviation of $0.15 \mathrm{~mm}$,

- the correctors follows the MQ within $0.2 \mathrm{~mm}$ of uncertainty, though statistics for correctors is much less.

Additional measurements will be performed as the spread of magnets movement is very close to the $0.15 \mathrm{~mm}$ tolerance.

\section{CONCLUSIONS}

The extensive cold tests and the analysis program of the arc SSS are being pursued with an increasing rate. Up to this point, all the magnets fulfilled the requested specifications for training performance. They all passed the nominal field gradient of 223 $\mathrm{T} / \mathrm{m}$ after the $2 \mathrm{nd}$ quench and reached the LHC ultimate field gradient of $241 \mathrm{~T} / \mathrm{m}$, after a maximum of six quenches. Warm measurements give a good evaluation of the geometric errors at cold apart from $b_{4}$ where the high spread in the warm/cold correlation is not yet understood. The multipoles at cold are consistent with the beam dynamics requirements apart from $\mathrm{b}_{6}$, which is for the first MQ above the allowed range. Measurements performed at warm on recent collared coils with the corrected cross-section show that $\mathrm{b}_{6}$ is now within the target [14]. The spread of the transfer function of the field gradient is above the allocated budget with an r.m.s. of 15 units. The origin could be an out of tolerance collar permeability [14]. This effect on the beam can however be reduced by appropriate pairing of the quadrupole in the accelerator lattice. The magnetic axes of the SSS (quadrupole and correctors) were measured on 10 SSS at cold and at warm. The magnet movements during the cool down are close to the tolerances, preventing to start immediately a sampling of the cold measurements axes.

\section{ACKNOWLEDGMENT}

The authors wish to thank the teams in Industry, at the CEA Saclay and at CERN involved in the construction and testing of the SSS, in particular the teams who built and maintain the test equipment, perform the cold tests operation and analyze the results.

\section{REFERENCES}

[1] “The LHC main ring," LHC Design Report Vol. 1, CERN-2004-003, June 2004.

[2] T. Tortschanoff et al. " "The short straight sections for the LHC," in Proc. PAC97, Vancouver, Canada, 1997.

[3] M. Peyrot et al., "Construction of new prototype of main quadrupole cold masses for the arc short straight sections for the LHC," in Proc. MT16, Ponte Vedra Beach, FL, USA, 1999.

[4] L. Nielsen et al., "A modular design for the 56 variants of the short straight sections in the arcs for the Large Hadron Collider," in Proc. MT17, Geneva, Switzerland, 2001.

[5] R. Burgmer et al., "Launching of series fabrication of the LHC main quadrupoles," in Proc. MT17, Geneva, Switzerland, 2001.

[6] V. Chohan et al., "Testing of the LHC magnets in cryogenic conditions, current experience and near future outlook," in Proc. EPAC 2004, Lucerna, Switzerland, 2004.

[7] L. Bottura et al., "A system for series magnetic measurements of the LHC main quadrupoles," IEEE Trans. Appl. Sup., vol. 12, no. 1, pp. 168-169, 2002.

[8] J. DiMarco et al., "Field alignment of quadrupole magnets for the LHC insertion regions," IEEE Trans. Appl. Sup., vol. 10, no. 1, pp. 127-130, 2000.

[9] J. Billan et al., "Twin rotating coils for cold magnetic measurements of $15 \mathrm{~m}$ long LHC dipoles," IEEE Trans. Appl. Supercond., vol. 10, no. 1, pp. 1422-1426, 2000.

[10] A. Lombardi, "Updated error tolerances for the main quadrupoles," CERN Memorandum, EDMS Number 498049, Mar. 2004.

[11] M. Peyrot et al., "Analysis of warm magnetic measurements of the first series-design prototypes of the LHC main quadrupoles," in Proc. EPAC 2000, Vienna, Austria, 2000.

[12] L. Bottura, Computation performed with Maelstrom software.

[13] R. Wolf and S. Le Naour, "The expected persistent current field errors in the LHC main dipole and quadrupole," CERN, LHC project note-230, 2000.

[14] E. Todesco, "Bimonthly report on field quality of quadrupoles June-July 2004," CERN, EDMS number 459 177, Sep. 2004. 